\title{
Les débuts de la spintronique Travaux précurseurs et magnétorésistance géante
}

Albert Fert (albert.fert@thalesgroup.com)

Unité Mixte de Physique CNRS/Thales, 91767 Palaiseau, et Université Paris-Sud, 91405 Orsay

Prix Nobel de Physique 2007

Dans cet article, Albert Fert

présente les débuts

et les concepts fondamentaux

de la spintronique :

les premiers travaux effectués

à Orsay sur la conduction

électrique des alliages

ferromagnétiques, les progrès

dans l'élaboration des couches

minces, la découverte

de la magnétorésistance

géante en 1988, et les

résultats importants obtenus

au début des années 90 ,

qui ont permis de

déboucher sur de

nombreuses applications.

Dans une seconde partie,

il traite plus en détail de

la physique de l'accumulation

de spins, qui gouverne

la propagation d'un courant

polarisé de spin

dans une succession

de conducteurs magnétiques

et non magnétiques.
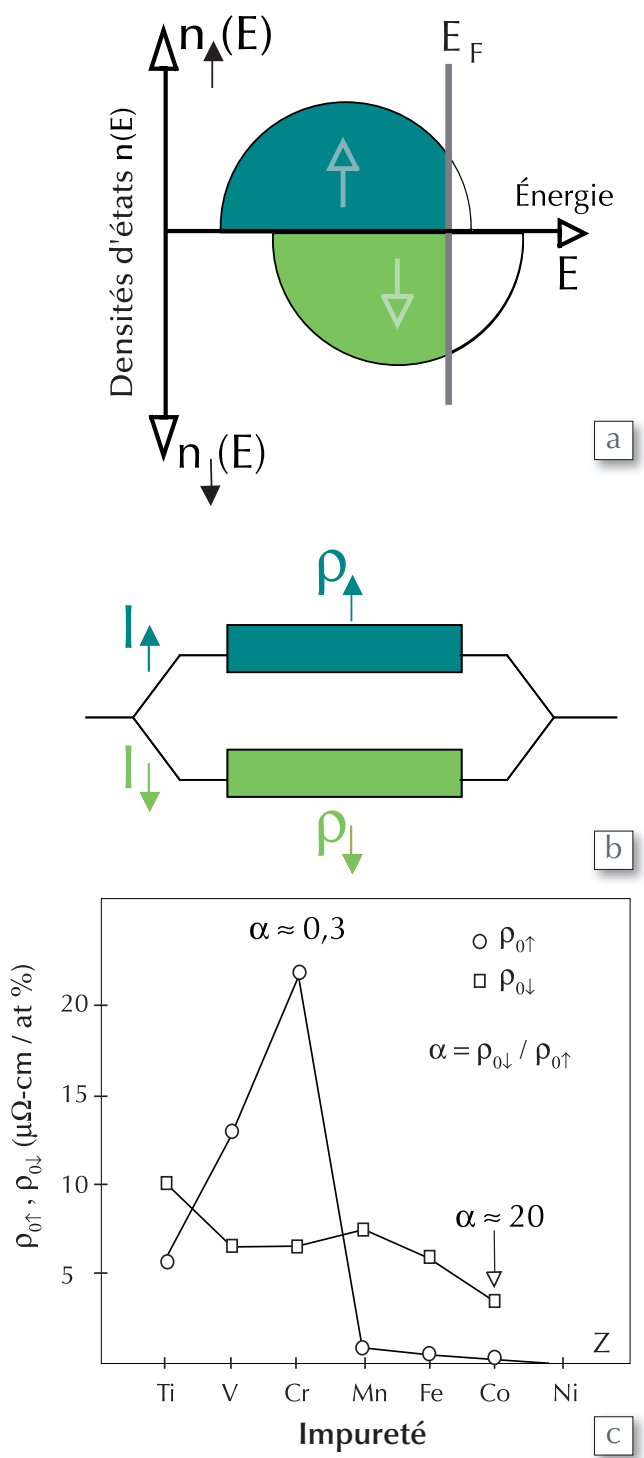

1. (a) Représentation schématique des densités d'états $\mathrm{n}(\mathrm{E})$ des bandes d'énergie décalées pour les électrons de spin majoritaire (spin $\uparrow$ en bleu) et minoritaire (spin $\downarrow$ en vert) dans un métal ferromagnétique. (b) Illustration de la conduction par deux canaux indépendants de résistivités $\rho_{\uparrow}$ et $\rho_{\downarrow}$ pour les électrons de spin $\uparrow$ et spin $\downarrow$ respectivement. Selon le métal, l'on peut avoir $\rho_{\downarrow}>\rho_{\uparrow}$ ou $\rho_{\downarrow}<\rho_{\uparrow}$ pour les résistivités des deux canaux. (c) Résistivités des canaux de spin $\uparrow$ et spin $\downarrow$ pour du nickel dopé avec $1 \%$ de différentes impuretés (mesures à 4,2 K) [4]. Dans un métal, la présence d'impuretés freine le courant et, à basse température, est l'origine principale de la résistivité électrique. Dans le cas d'un métal ferromagnétique, une impureté peut freiner très différemment les électrons de spin $\uparrow$ et spin $\downarrow$.
La spintronique peut se décrire comme une électronique qui exploite non seulement la charge, mais aussi le spin des électrons. Son développement a suivi la découverte de la magnétorésistance géante (GMR) en 1988 [1,2]. Le concept général de la spintronique est de placer des matériaux ferromagnétiques sur le trajet des électrons et d'utiliser l'influence du spin sur la mobilité des électrons dans ces matériaux. Cette influence, d'abord suggérée par Mott [3] en 1936, a été ensuite démontrée expérimentalement et décrite théoriquement à la fin des années 60 [4, 5]. La découverte de la GMR a conduit aux premières utilisations pratiques de cette influence. De nombreux autres phénomènes exploitant aussi le spin des électrons se sont ensuite révélés et, aujourd'hui, la spintronique se développe dans de très nombreuses directions, qui seront traitées dans les autres articles de ce dossier : magnétorésistance tunnel, phénomènes de transfert de spin, spintronique avec semi-conducteurs, spintronique moléculaire, spintronique avec multiferroïques, etc.

\section{La conduction électrique dans les métaux et alliages ferromagnétiques}

Un métal ferromagnétique, comme le fer ou le nickel, se caractérise par un décalage des bandes d'énergie des électrons de spin parallèle et antiparallèle à l'aimantation (fig. 1a). Ces bandes sont peuplées jusqu'au niveau de Fermi $\left(E_{F}\right.$ sur la figure $\left.1 a\right)$, et du décalage résultent des populations différentes pour les deux directions de spin, que nous appellerons dans la suite de l'article spin $\uparrow$ pour les spins majoritaires et spin $\downarrow$ pour les minoritaires. C'est l'origine de l'aimantation spontanée des ferromagnétiques. La conduction électrique, quant à elle, est liée à la mobilité des électrons à $\mathrm{E}_{\mathrm{F}}$, et du décalage des bandes résulte de façon générale une mobilité différente pour spin $\uparrow$ et spin $\downarrow$, ce qui, en fin de compte, se traduit par une conduction en parallèle par deux canaux de résistivités différentes (fig. 1b). L'influence du spin sur la conduction électrique dans les métaux ferromagnétiques fut proposée 
$\gg>$

par Mott [3] en 1936, pour expliquer certaines anomalies de la résistivité de ces métaux au voisinage de la température de Curie.

Cependant, à l'époque (en 1966) où j'arrivai au Laboratoire de Physique des Solides d'Orsay, le sujet était resté complètement inexploré et Ian Campbell me le proposa comme sujet de thèse. Cette thèse démarra donc par des mesures de la résistivité de fer et de nickel dopés de divers types d'impuretés. L'analyse comparée de la dépendance en température de la résistivité de ces alliages, ainsi que l'étude d'alliages ternaires que je décrirai plus loin, nous permirent de confirmer la proposition de Mott et de montrer que, pour certains dopages, la mobilité pouvait être très différente pour les électrons de spin $\uparrow$ et de spin $\downarrow$. La figure 1c montre les valeurs des résistivités des deux canaux pour du nickel contenant $1 \%$ de diverses impuretés [4]. On peut voir que le rapport $\alpha$ entre les résistivités $\rho_{0 \downarrow}$ et $\rho_{0 \uparrow}$ des canaux de spin $\downarrow$ et de spin $\uparrow$ peut être aussi grand que 20 pour des impuretés de cobalt, mais aussi être plus petit que 1 pour des impuretés de chrome ou de vanadium.

Ces résultats pouvaient être expliqués par les modèles de structure électronique développés par Friedel pour les alliages de métaux ferromagnétiques. Après les premières mesures de la fin des années 60 , le modèle de conduction à deux courants dans les métaux et alliages ferromagnétiques fut rapidement confirmé dans d'autres groupes, par exemple par Loegel et Gautier [5] dans les cas d'alliages de cobalt.

À la fin de ce premier chapitre, je voudrais faire remarquer que la spintronique n'a retenu jusqu'à présent qu'une version simplifiée du modèle à deux courants de ma thèse, en oubliant en route les effets de spin mixing [4], c'est-à-dire les échanges de quantité de mouvement par renversement de spin, essentiellement par collision entre électrons et ondes de spin dans un métal ferromagnétique. La " résistivité de spin mixing ", $\rho_{\uparrow \downarrow}$, augmente progressivement avec la température. À la température ambiante, elle atteint une dizaine de $\mu \Omega-\mathrm{cm}$ dans des métaux comme le fer ou le nickel, et égalise partiellement les deux courants. De nombreux résultats de spintronique, pour une analyse plus rigoureuse, devraient sans doute être revisités en tenant compte du spin mixing.
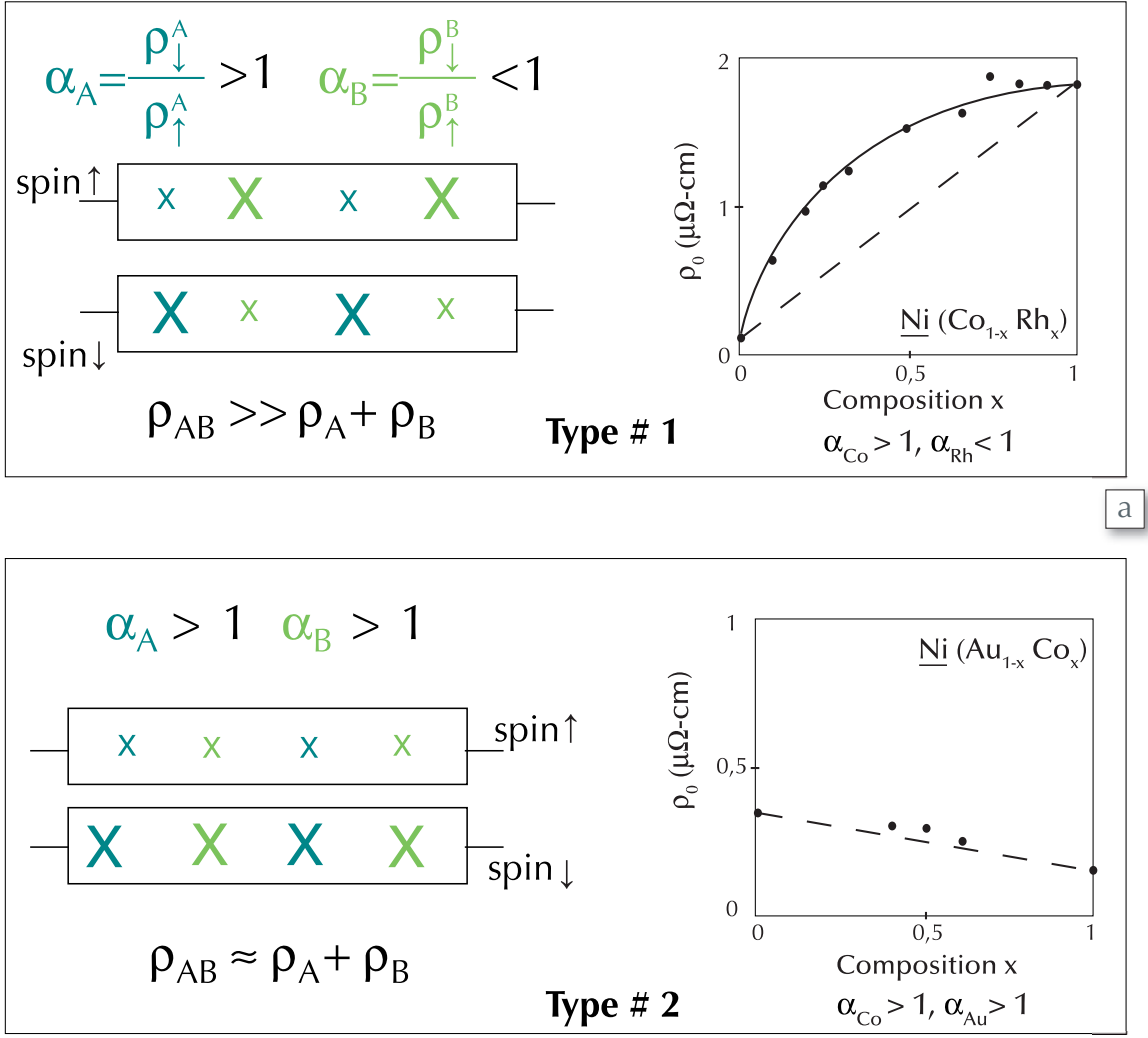

b

2. Expériences sur des alliages ternaires, basées sur le même concept que la GMR [4]. Sur les schémas illustrant la conduction par deux canaux, on a représenté l'efficacité plus ou moins grande d'une impureté A (bleu) ou B (vert) au freinage des électrons par des X (représentatifs d'obstacles) plus ou moins grands. (a) Schéma pour la conduction dans des alliages dopés avec des impuretés diffusant les électrons avec des asymétries en spin opposées $\left(\alpha_{A}=\rho_{A \downarrow} / \rho_{A \uparrow}>1, \alpha_{B}=\rho_{B \downarrow} / \rho_{B \uparrow}<1, \rho_{A B}>>\rho_{A}+\rho_{B}\right)$ et résultats expérimentaux pour des alliages $\mathrm{Ni}\left(\mathrm{C}_{1-x} \mathrm{Rh} \mathrm{h}_{x}\right)$ [4]. (b) Même schéma qu'en (a) pour des alliages dopés avec des impuretés diffusant les électrons avec des asymétries en spin semblables $\left(\alpha_{A}>1, \alpha_{B}>1, \rho_{A B} \approx \rho_{A}+\rho_{B}\right)$ et résultats expérimentaux pour des alliages $\mathrm{Ni}\left(\mathrm{Au}_{1-\mathrm{x}} \mathrm{C} \mathrm{O}_{\mathrm{x}}\right)$. Pour la GMR, les impuretés $\mathrm{A}$ et $\mathrm{B}$ sont remplacées par des couches magnétiques $\mathrm{F} 1$ et $\mathrm{F} 2$ d'un même métal ferromagnétique, la situation de a (b) correspondant à la configuration antiparallèle (parallèle) des aimantations des couches F1 et F2.
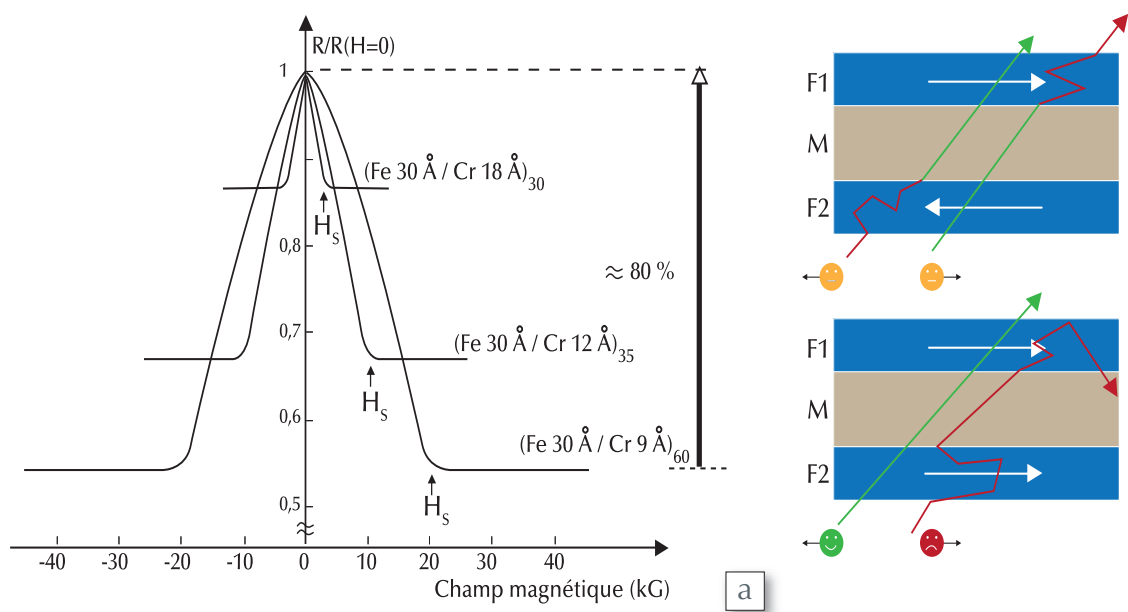

3. (a) Premières observations de magnétorésistance géante à Orsay sur des multicouches $\mathrm{Fe} / \mathrm{Cr}(001)$ [1]. La résistance, mesurée pour un courant parallèle aux couches, chute d'une valeur $R_{A p}$ à une valeur $R_{p}$ quand un champ magnétique aligne les aimantations. Avec la définition adoptée aujourd'hui pour le rapport de magnétorésistance, $M R=100\left(R_{A p}-R_{p}\right) / R_{p}$, MR vaut $80 \%$ pour la multicouche ( Fe $\left.3 n m / C r ~ 0,9 n m\right)$.

(b) Schéma du mécanisme de la GMR. Dans la configuration magnétique parallèle P (bas), les électrons d'une direction de spin peuvent traverser facilement toutes les couches magnétiques et le court-circuit par ce canal conduit à une faible valeur de la résistance. Dans la configuration antiparallèle AP (haut), les électrons de chaque canal sont ralentis une fois sur deux en traversant les couches magnétiques, et la résistance est élevée. Les trajectoires des électrons sont représentées obliques car, même pour un courant parallèle aux couches, c'est seulement la direction moyenne des vitesses qui est parallèle aux couches. On passe de la configuration antiparallèle AP à la configuration parallèle P par application d'un champ magnétique. 


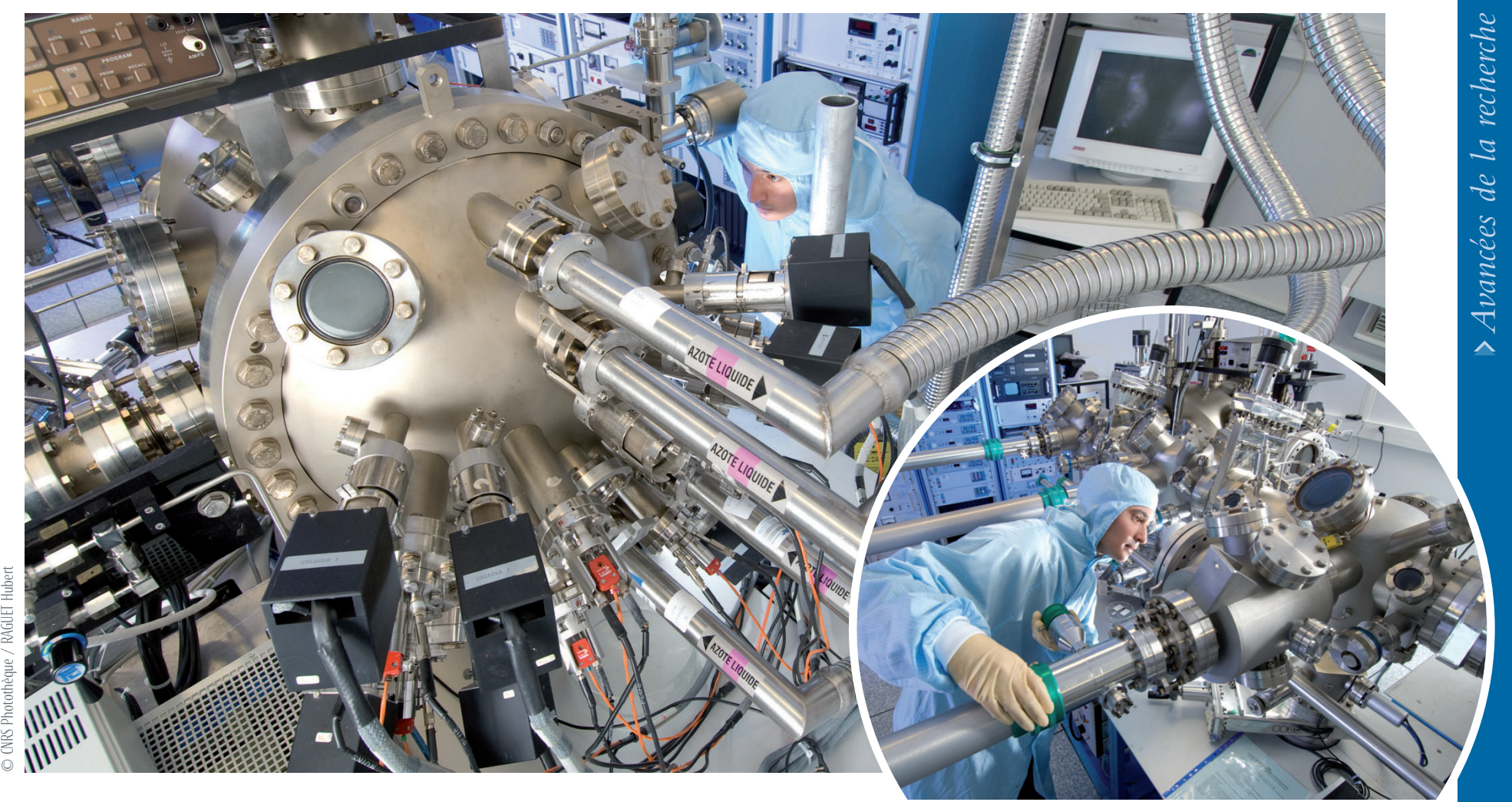

Chambre d'épitaxie par jets moléculaires utilisée pour la croissance de multicouches magnétiques (UMR 137, Unité mixte de physique CNRS/THALES, Orsay). Ce dispositif permet le dépôt sur un substrat de couches métalliques ultraminces, de quelques plans atomiques d'épaisseur. l'empilement alterné de films minces magnétiques et non magnétiques permet d'obtenir des multicouches magnétiques dans lesquelles est produit l'effet de magnétorésistance géante.

\section{Le concept de la magnétorésistance géante dans des expériences sur des alliages magnétiques ternaires}

Dans mon travail avec Ian Campbell, certaines expériences sur des alliages ternaires [4] anticipaient déjà la GMR, comme illustré par la figure 2. Supposons, par exemple, que l'on dope du nickel à la fois avec des impuretés de Co, qui freinent fortement les électrons dans le canal de spin $\downarrow$ en laissant le canal de spin $\uparrow$ relativement " ouvert ", et des impuretés de Rh pour lesquelles c'est l'inverse. Dans l'alliage $\mathrm{Ni}(\mathrm{Co}+\mathrm{Rh})$, que nous appellerons de type \#1, les électrons des deux canaux sont alors fortement freinés, soit par les impuretés de Co dans un canal, soit par celles de Rh dans l'autre. Il n'y a plus de canal "ouvert ", et la résistivité est fortement augmentée par rapport à celle d'alliages contenant seulement soit Co, soit Rh (fig. 2a). Au contraire, il n'y a pas cette augmentation de résistivité pour des alliages de type \#2 dopés par des impuretés (Co et Au, par exemple) qui, toutes deux, freinent les électrons d'un même canal et laissent le deuxième canal relativement libre (fig. 2b).

L'idée de la GMR est de remplacer les impuretés A et B de l'alliage ternaire par deux couches magnétiques F1 et F2 d'un même métal ferromagnétique, séparées par une couche non magnétique $M$ (fig. 3b). Si les deux couches ont leurs aimantations dans des directions opposées (configuration dite antiparallèle, AP), la couche F1 freinera les électrons d'un canal, la couche F2 ceux de l'autre canal, et l'on retrouve donc la situation de résistivité élevée d'un alliage ternaire de type \#1 dans lequel les électrons des deux canaux sont freinés, par les impuretés A dans l'un, les impuretés B dans l'autre. Par contre, la configuration d'aimantations parallèles $(\mathrm{P})$ correspond à l'alliage de faible résistivité de type \#2 dans lequel les impuretés A et B laissent un des canaux relativement libre. L'élément nouveau vient de la possibilité de passer de grande à petite résistivité, en appliquant un champ magnétique pour aligner les aimantations d'une configuration initialement antiparallèle.

Cependant, les équations de transport nous disent que les électrons ne pourront sentir l'orientation relative des aimantations des couches F1 et F2 que si la distance entre les couches est inférieure au « libre parcours moyen " des électrons, c'est-à-dire, en pratique dans des films métalliques, si cette distance n'est que de quelques nanomètres. Malheureusement, en 1970, il n'était pas techniquement possible de fabriquer des multicouches constituées de couches épaisses de seulement quelques nanomètres. Il fallut attendre le milieu des années 80 .

\section{La découverte de la GMR}

Au milieu des années 80, avec le développement de techniques de dépôt sous ultravide comme l'épitaxie par jets moléculaires (EJM), il devint possible de fabriquer des multicouches empilant des couches ultrafines et d'envisager l'extension des expériences de ma thèse sur des alliages ternaires à des multicouches magnétiques. De plus, en 1986, les expériences de diffusion Brillouin de Peter Grünberg [6] révélèrent l'existence d'un " couplage d'échange " antiferromagnétique entre couches de fer ferromagnétiques, séparées par de très fines couches de chrome non magnétiques. Une multicouche $\mathrm{Fe} / \mathrm{Cr}$ pouvait donc donner la possibilité de commuter d'une configuration à aimantations opposées à une autre à aimantations parallèles, par application d'un champ magnétique. À cette époque, j’avais justement discuté de l'intérêt des multicouches magnétiques avec Alain Friederich du LCR Thomson-CSF, et une collaboration s'était établie entre mon équipe d'Orsay et la sienne pour l'élaboration de telles couches. Patrick Étienne, l'expert EJM au LCR, adapta aux métaux les techniques mises au point pour les semi-conducteurs et guida les premières fabrications de multicouches $\mathrm{Fe} / \mathrm{Cr}$ de la thèse de Frédéric Nguyen Van Dau. Cela conduisit rapidement à nos premières observations (fig. 3a) de la GMR 
$\gg>$

sur des super-réseaux $\mathrm{Fe} / \mathrm{Cr}(001)$ au début de 1988 [1]. Les postdocs Mario Baibich et Jean-Marc Broto, ainsi que les doctorants Agnès Barthélémy et Frédéric Petroff, participèrent aussi à l'aventure. Des résultats semblables sur des tricouches $\mathrm{Fe} / \mathrm{Cr} / \mathrm{Fe}$ furent obtenus à peu près simultanément dans l'équipe de Peter Grünberg à Jülich [2]. Les équipes française et allemande déposèrent des brevets sur les applications possibles de la GMR ; les Allemands furent les plus rapides.

L'interprétation de la GMR est en gros semblable à celle des expériences sur des alliages ternaires que nous avons décrites plus haut, et est schématisée dans la figure $3 b$. Un modèle classique de la GMR fut publié dès 1989 par Camley et Barnas [7]. Vint ensuite un modèle quantique que je publiai avec Levy et Zhang en 1991 [8].

\section{l'âge d'or de la GMR}

La découverte de la GMR attira rapidement l'attention, aussi bien pour ses possibilités d'applications que pour l'intérêt fondamental d'expériences de transport électronique exploitant le spin. Les études de la GMR se multiplièrent dans de nombreux laboratoires et notre petite équipe se lança aussi avec enthousiasme dans l'exploration de ce nouveau champ de recherche, qui allait s'appeler bientôt "spintronique ".

Sur le plan expérimental, deux résultats importants furent publiés en 1990. Stuart Parkin et al [9] démontrèrent l'existence de GMR dans des multicouches $\mathrm{Fe} / \mathrm{Cr}$, $\mathrm{Co} / \mathrm{Ru}$ et $\mathrm{Co} / \mathrm{Cr}$ élaborées par la méthode plus simple et plus rapide de pulvérisation cathodique. Ils découvrirent aussi une oscillation de la GMR en fonction de l'épaisseur des couches d'espacement ( $\mathrm{Cr}$ ou $\mathrm{Ru}$ ). Cette oscillation traduit la succession périodique d'épaisseurs dans lesquelles l'interaction entre couches magnétiques est antiferromagnétique (ce qui donne de la GMR) et d'épaisseurs à interaction ferromagnétique (sans GMR).

Également en 1990,l'équipe de Jean-Pierre Renard à Orsay et celle de Terua Shinjo à Kyoto démontrèrent que les effets GMR pouvaient aussi être obtenus dans des multicouches sans couplage d'échange antiferromagnétique, mais composées de couches magnétiques de coercivités différentes $[10,11]$. Un autre résultat important, en 1991, fut aussi l'observation d'effets GMR grands et oscillants dans $\mathrm{Co} / \mathrm{Cu}$, qui deviendra un archétype de système GMR. Les premières observations [12] furent
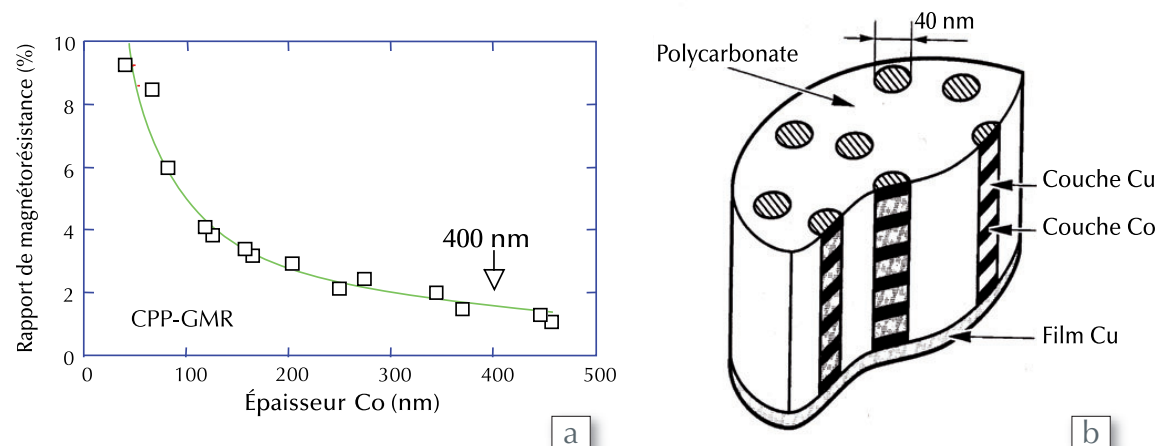

4. (a) Variation de la GMR en courant perpendiculaire aux couches (CPP-GMR) de nanofils multicouches Co/Cu (8nm) en fonction de l'épaisseur des couches de cobalt [19]. La GMR subsiste jusqu'à des épaisseurs de l'ordre de grandeur du micron. (b) Structure des nanofils multicouches utilisés pour les mesures de (a).

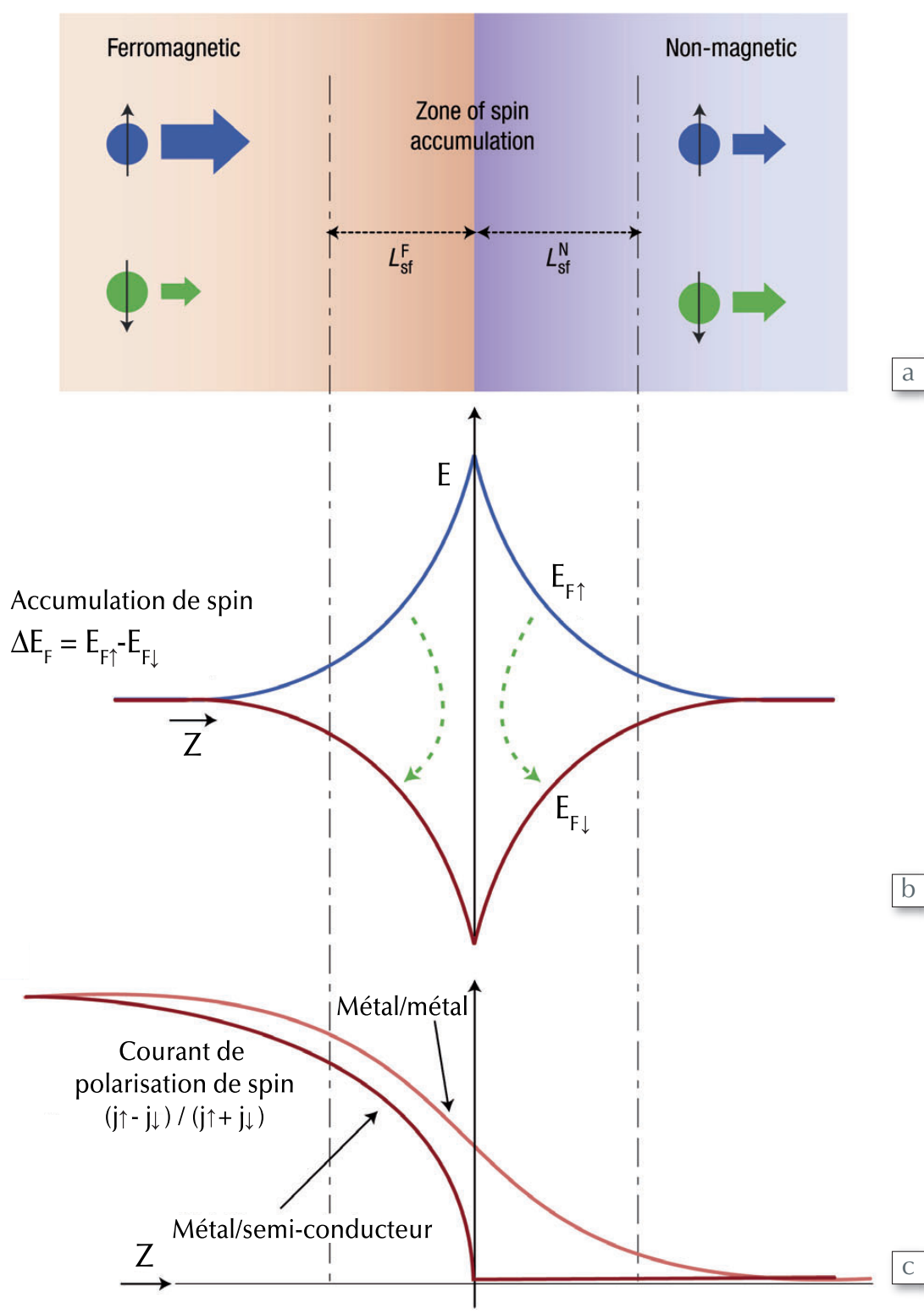

5. Représentation schématique de l'accumulation des spins, associée au passage d'un courant d'un conducteur ferromagnétique à un conducteur non magnétique.

(a) : Flux respectifs d'électrons de spin $\uparrow$ (flèches bleues) et de spin $\downarrow$ (flèches vertes) loin de l'interface.

$L_{s f}^{F}$ et $L_{s f}^{N}$ sont les longueurs de diffusion de spin dans les matériaux ferromagnétique et non magnétique, respectivement. (b) : Variation des niveaux de Fermi $E_{\uparrow \uparrow}$ et $E_{F_{\downarrow}}$ au voisinage de l'interface ; les flèches symbolisent les renversements de spin générés par l'accumulation.

(c) : Variation de la polarisation en spin du courant. 
réalisées par un doctorant brésilien de notre équipe, Dante Mosca, sur des multicouches préparées par pulvérisation cathodique à Michigan State University, et, à peu près à la même époque, dans l'équipe de Stuart Parkin.

En 1991 également, Bernard Dieny et l'équipe d'IBM Almaden [13] montrèrent la possibilité de détecter de très petits champs magnétiques par GMR dans des tricouches dites spin valves, aujourd'hui à la base de nombreux dispositifs $[14,15]$. Les spin valves et leurs applications seront décrites dans les autres articles de ce dossier.

Parallèlement à l'exploration de la GMR dans de nombreux types de structures, un gros effort de recherche fut fait pour le développement de modèles théoriques, et notamment de modèles s'appuyant sur des déterminations ab initio de la structure électronique des multicouches. Une description de ces modèles serait trop longue, et le lecteur pourra consulter des articles de revue récents [16].Je veux cependant faire remarquer que l'influence du spin sur la conduction électrique, et la GMR en particulier, est très dépendante de défauts difficiles à connaître précisément (impuretés, rugosité d'interface, etc.). Les modèles théoriques ne peuvent guère prendre en compte ces défauts de façon réaliste et ne sont pas réellement prédictifs. La situation est différente pour la GMR en courant perpendiculaire aux couches, décrite plus loin.

Les applications de la GMR seront présentées par J.P. Nozières et C. Fermon dans ce même dossier. L'application la plus connue est la lecture des disques durs $[14,15]$, où les capteurs GMR à structure spin valve ont remplacé la magnétorésistance classique. La GMR, grâce à sa sensibilité et aux possibilités amenées pour la miniaturisation des capteurs, a rendu possible une forte réduction de la taille des inscriptions magnétiques et a ainsi conduit à une augmentation de la capacité d'information stockée dans les disques, de plus de deux ordres de grandeur (de $\approx 1$ à $\approx 600$ Gbit/in ${ }^{2}$ en 2009). Cette augmentation a conduit non seulement à la réalisation de disques de très grande capacité (jusqu'au teraoctet), mais aussi à celle de disques de la taille de pièces de monnaie pour l'électronique nomade, baladeurs, appareils photographiques, etc. Les capteurs GMR ont aussi des applications dans l'industrie automobile et les technologies biomédicales.

\section{La GMR en courant perpendiculaire aux couches}

Pendant les premières années de recherche sur la GMR, les expériences sur les multicouches étaient effectuées avec un courant électrique orienté dans le plan des couches, dans la géométrie que l'on appelle maintenant CIP (Current In Plane). Les premières expériences en géométrie CPP (Current Perpendicular to the layer Planes) furent réalisées par l'équipe de Jack Bass et al. [17] à Michigan State University, en plaçant une multicouche en sandwich entre deux couches supraconductrices de niobium, qui établissent des plans équipotentiels sur les deux faces de la multicouche et permettent d'obtenir un courant perpendiculaire aux couches.

En CPP, la GMR est non seulement nettement plus grande qu'en CIP (la CPP-GMR sera d'ailleurs utilisée dans la prochaine génération de têtes de lecture de disques durs), mais aussi subsiste pour des couches relativement épaisses, jusqu'au voisinage du micron, comme on peut le voir sur la figure 4 pour des multicouches élaborées par électrodéposition dans des pores de membranes de polycarbonates [18]. Le modèle Valet-Fert [19] permet d'expliquer cette différence par les effets d'accumulation de spin spécifiques de la situation CPP. La longueur d'échelle des phénomènes de transport devient la longueur de diffusion de spin, nettement plus grande que le libre parcours moyen impliqué dans la géométrie usuelle.

\section{Physique de l'accumulation de spin}

En fait, la CPP-GMR a permis de comprendre la physique de l'accumulation de spin qui gouverne de façon très générale la propagation d'un courant polarisé de spin dans une succession de conducteurs magnétiques et non magnétiques, et joue un rôle important dans tous les développements actuels de la spintronique. Le courant de diffusion généré par l'accumulation de spin est le mécanisme permettant à un courant polarisé en spin de se propager loin de l'interface entre un matériau magnétique et un matériau non magnétique, au-delà de la longueur balistique, jusqu'à une distance de l'ordre de grandeur de la longueur de diffusion de spin. On verra dans l'encadré (p. 10) que ce transport de spin à grande distance a permis d'étendre les études de GMR à des structures latérales. Dans des nanotubes de carbone, par exemple, la longueur de diffusion de spin excède $20 \mu \mathrm{m}$, et des courants polarisés peuvent être transportés d'un bout à l'autre de nanotubes de carbone relativement longs [20].

Comme expliqué sur la figure 5, une accumulation de spin se produit quand un flux d'électrons passe d'un matériau ferromagnétique à un conducteur non magnétique. Le flux incident est plus grand dans un des canaux (spin $\uparrow$ sur la figure), alors que le flux sortant est également réparti (fig. 5a). Au voisinage de l'interface, il y a donc accumulation d'électrons d'une certaine direction de spin (spin $\uparrow$ sur la figure) et, neutralité de charge oblige, déplétion de l'autre direction

\section{Références}

1 - M.N. Baibich, J.M. Broto, A. Fert, F. Nguyen Van Dau, F. Petroff, P. Etienne, G. Creuzet, A. Friederich, J. Chazelas, Phys. Rev. Lett. 61 (1988) 2472.

$2 \bullet$ G. Binash, P. Grünberg, F. Saurenbach, W. Zinn, Phys. Rev. B 39 (1989) 4828.

3 • N.F. Mott, Proc. Roy. Soc. A 153 (1936) 699

4 - A. Fert et I. A. Campbell, Phys. Rev. Lett. 21 (1968) 1190, J. Physique 32 (1971) (1-46, J. Phys. F 6 (1976) 849.

5 - B. Loegel et F. Gautier, J. Phys. Chem. Sol. 32 (1971) 2723.

6•P. Grünberg et al., Phys. Rev. Lett. 57 (1986) 2442.

7 - R. E. Camley et J. Barnas, Phys. Rev. Lett. 63 (1989) 664.

8 - P.M. Levy, S. Zhang, A. Fert, Phys. Rev. Lett. 65 (1990) 1643.

9 - S.S.P. Parkin, N. More, K.P. Roche, Phys. Rev. Lett. 64 (1990) 2304.

10 • T. Shinjo et H. Yamamoto, J. Phys. Soc. Jpn. 59 (1990) 3061.

11 • C. Dupas et al., J. Appl. Phys. 67 (1990) 5680.

12 • D. H. Mosca et al., J. Magn. Magn. Mater. 94 (1991) L1 ; S. S. P. Parkin et al., Phys. Rev. Lett. 66 (1991) 2152.

13 • B. Dieny et al, Phys. Rev. B 43 (1991) 1297.

14 - S.S.P. Parkin, dans Spin Dependent Transport in Magnetic nanostructures (édité par S. Maekawa et T. Shinjo, Taylor and Francis 2002), p. 237.

15 - C. Chappert, A. Fert, F. Nguyen Van Dau, Nature Materials, 6 (2007) 813.

16 • T. Shinjo, ibidem ref. [14], p. 1 P. M. Levy et I. Mertig, ibidem, p. 47 A. Fert, A. Barthélémy et $A$. Fert dans Nanomagnetism (édité par D.L. Mills et J.A.C. Bland, Elsevier, 2006), p. 153.

17 - W. P. Pratt et al., Phys. Rev. Lett. 66 (1991) 3060 ; J. Bass et W. P. Pratt, J. Magn. Magn. Mater. 200 (1999) 274.

18 • L. Piraux et al., Appl. Phys. Lett. 65 (1994) 2484 ; A. Fert et L. Piraux, J. Magn. Magn. Mater. 200 (1999) 338.

19 • T. Valet et A. Fert, Phys. Rev. B 48 (1993) 7099

20 • L.H. Hueso et al., Nature 445 (2007) 410.

21 - B. T. Jonker et M. E. Flatté, dans Nanomagnetism (édité par D.L. Mills et J.A.C. Bland, Elsevier, 2006), p. 227. 
de spin, ou, en d'autres termes, décalage entre les niveaux de Fermi (potentiels chimiques) des deux directions de spin (fig. 5b). L'accumulation de spin diffuse des deux cotés de l'interface et décroît exponentiellement, pour s'étendre jusqu'à une distance de l'ordre de grandeur de la longueur de diffusion de spin. Cette distribution hors d'équilibre génère les renversements de spin, qui ajustent les flux entrant et sortant. Pour résumer, il y a au voisinage de l'interface une large zone d'accumulation de spin, dans laquelle le courant polarisé de spin venant du conducteur magnétique est progressivement dépolarisé par les renversements de spin générés par l'accumulation de spin (fig. 5c).

La figure 5 représente le cas de l'injection de spin, c'est-à-dire d'électrons allant du magnétique au non magnétique. Pour des électrons allant en direction opposée (extraction de spin), on a une situation semblable, mais avec accumulation de la direction de spin opposée et une polarisation progressive du courant par les renversements de spin qui en résultent. Dans les deux cas, injection ou extraction, la polarisation du courant subsiste ou commence loin de l'interface dans le matériau non magnétique. Cette physique peut se décrire par des équations de transport [19], dans lesquelles le potentiel électrique est remplacé par un potentiel électrochimique dépendant du spin. La physique de l'accumulation de spin joue un rôle important dans la plupart des développements récents de la spintronique. Ses équations peuvent être appliquées non seulement au cas simple d'une interface isolée, mais aussi à la situation multi-interfaces avec interaction entre les accumulations de spin à des interfaces voisines. Elles peuvent aussi être généralisées à la situation des structures avec semi-conducteurs ou molécules, prendre en compte la courbure de bande à l'interface de semi-conducteurs, ou encore les configurations magnétiques non colinéaires des expériences de transfert de spin (cf. article de B. Diény dans ce dossier).

\section{Conclusion}

La découverte de la GMR résulte de la rencontre entre des progrès technologiques et une base de physique fondamentale, décrivant l'influence du spin sur le transport électronique dans les matériaux ferromagnétiques. On réalisa rapidement quelles applications importantes on pouvait tirer de l'exploitation simultanée de la charge et du spin des électrons, et cela déclencha une recherche active, qui révéla d'autres phénomènes exploitant également l'influence du spin en électronique. La spintronique se développe maintenant sur de nombreux nouveaux axes et sera très certainement à la base d'applications technologiques dans de multiples domaines.

\section{- La magnétorésistance géante dans des structures « latérales "}

Le cas d'une structure "latérale » de type source/canal latéral/drain, composée d'un conducteur non magnétique $\mathrm{N}$ dont les extrémités sont contactées à des conducteurs ferromagnétiques source $\mathrm{F} 1$ et drain F2, dont les aimantations peuvent être parallèles ou antiparallèles (voir exemple de structure sur la figure 6 a et b), est semblable à celui de la CPP-GMR. Le courant passe latéralement de $\mathrm{F} 1$ à $\mathrm{N}$, puis à $\mathrm{F} 2$, de la même façon qu'il traverserait verticalement en CPP-GMR une tricouche, avec une couche non magnétique dont l'épaisseur serait égale à la longueur du canal et encadrée par deux couches magnétiques semi-infinies. La structure latérale a l'avantage de rendre possible l'installation d'une " grille» sur le canal.

Les résultats sur ces structures latérales s'interprètent avec le même type d'équations de drift-diffusion que celles de la CPPGMR. Dans une situation de CPP-GMR, avec une épaisseur de couche non magnétique égale à la longueur du canal latéral, donc en général supérieure à 100 nm, et avec les métaux habituels de la GMR, les performances en termes de variation de résistance $\Delta R$ entre configurations parallèle et antiparallèle ou de signal de sortie $\Delta V$ restent cependant très modestes : soit $\Delta R / R$ ou $\Delta V / V_{\text {bias }}$ de l'ordre de quelques pourcents ou dixièmes de pourcents, avec des contacts tunnel. Quand le canal latéral est un semiconducteur, qui peut certes avoir un temps de relaxation de spin plus long qu'un métal, mais par contre une vitesse électronique beaucoup plus petite, les performances sont encore plus modestes [21].

Obtenir des performances élevées avec des structures latérales exige donc des matériaux combinant des temps de relaxation de spin longs, mais aussi des vitesses électroniques grandes, nanotubes de carbone ou graphène, par exemple. Un exemple est donné sur la figure $6 \mathrm{c}$ et $\mathrm{d}$, avec des résultats de magnétorésistance supérieurs à $70 \%$ et des signaux de sortie $\mathrm{V}$ allant jusqu'à $50 \mathrm{mV}$, dans des expériences où le canal latéral est un nanotube de carbone [20].
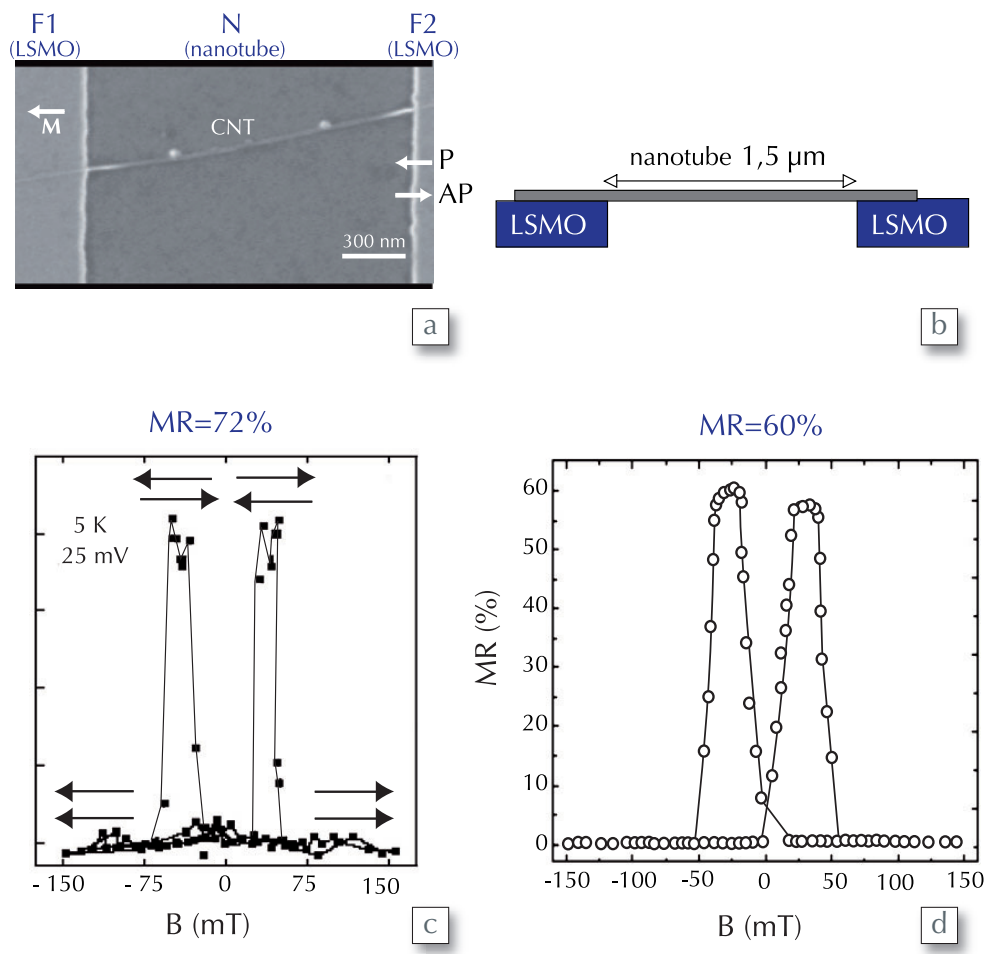

6. (a) Image par microscopie électronique d'un nanotube de carbone (CNT) entre contacts (source et drain) de $\mathrm{La}_{2 / 3} \mathrm{Sr}_{1 / 3} \mathrm{MnO}_{3}$ (LSM0). L'aimantation M de la source F1 est fixe; celle du drain F2 peut être parallèle (P) ou antiparallèle $(A P)$ à $M$. (b) Schéma correspondant (vue latérale). (c) et (d) : Magnétorésistances de $72 \%$ et $60 \%$, avec des structures du type (a) [20]. Sur la figure de gauche, les directions des aimantations des matériaux F1 et F2 sont indiquées par des flèches. On remarquera l'hystérésis en cyclage de champ, du au faible champ coercitif de la couche F1. 